\title{
Comparison of manual data coding errors in two hospitals
}

\author{
PA HALL, NR LEMOINE \\ From the Department of Morbid Anatomy, The London Hospital
}

SUMMARY The routine manual encoding of pathological data, using the SNOP and SNOMED systems at two London teaching hospitals, was reviewed. The error rates in the two departments were compared and the causes analysed. The relative merits of SNOP and SNOMED were considered. Methods to optimise the efficiency of manual encoding are suggested and the importance of accuracy in coding is emphasised.

A routine histopathology department produces a vast quantity of data, and it is important that such data are carefully recorded and suitably indexed, usually by numerical code. Storage and retrieval of the information has often entailed a card index, but computers are increasingly being used because of their speed, storage capacity, and their potential for data manipulation. Although the use of computers to perform the data encoding has been advocated, ${ }^{1-3}$ this is not widely used at present: it is more common for the reporting pathologist to perform the encoding manually, often using either the SNOP ${ }^{4}$ or SNOMED 5 systems. The accuracy of such manual encoding is clearly important, and it is surprising that there have been few studies on the magnitude and causes of errors in non-automated coding systems. ${ }^{6}$ The widespread adoption of computers in all branches of pathology may ease data handling but it cannot compensate for inaccurate data.

The departments of morbid anatomy at both the London Hospital and University College Hospital (UCH) use the same computerised report system, ${ }^{7}$ and both use a system of manual data encoding by the reporting pathologists. They differ in that $\mathrm{UCH}$ uses SNOMED while the London Hospital uses SNOP. The rationale for report coding is to allow cases to be retrieved for research series, teaching purposes, and for departmental auditing. The computerised records system permits the rapid collection of previous reports on material from each patient. As a result of our personal experience of the results of poor coding, we decided to perform a comparative study of error rates and their causes at both institutions.

Accepted for publication 23 January 1986

\section{Material and methods}

The same protocol was followed at both institutions: we scrutinised a consecutive series of 2500 reports from early 1985 . No other member of staff was aware of the sequence of records being examined until the study had been completed. The text of each report was examined and compared with the final typed numerical codes that were recorded. The codes on the handwritten original reports were compared with those on the typewritten report to assess the influence of handwriting and the magnitude of purely secretarial errors. The member of staff who performed the encoding was identified from the handwritten reports. Any case that contained an error of omission or comission was recorded by surgical number, diagnoses, appropriate code(s), recorded code(s), retrievability of error(s), and trainee or consultant pathologist.

The appropriate codes were selected from the SNOP or SNOMED reference texts and were agreed on by both authors. Diagnoses and specific histological features not found within the SNOP or SNOMED compendia were assigned new codes, which were in keeping with the hierarchical format of both systems. An ERROR was defined as any code that we both agreed was incorrect, unsuitable, or inappropriate for the final written report, or that had been omitted. For practical purposes this required the accurate encoding of all pathological diagnoses that appeared in the written report, irrespective of their perceived relative importance to the reporting pathologist. The computer retrieval of data is objective and cannot distinguish between "incorrect" and "nearly correct"; it depends on the codes assigned, which must also be objective and accurate. For the purposes of this study the errors were divided into two groups. An IRRETRIEVABLE ERROR was defined as "an error that 


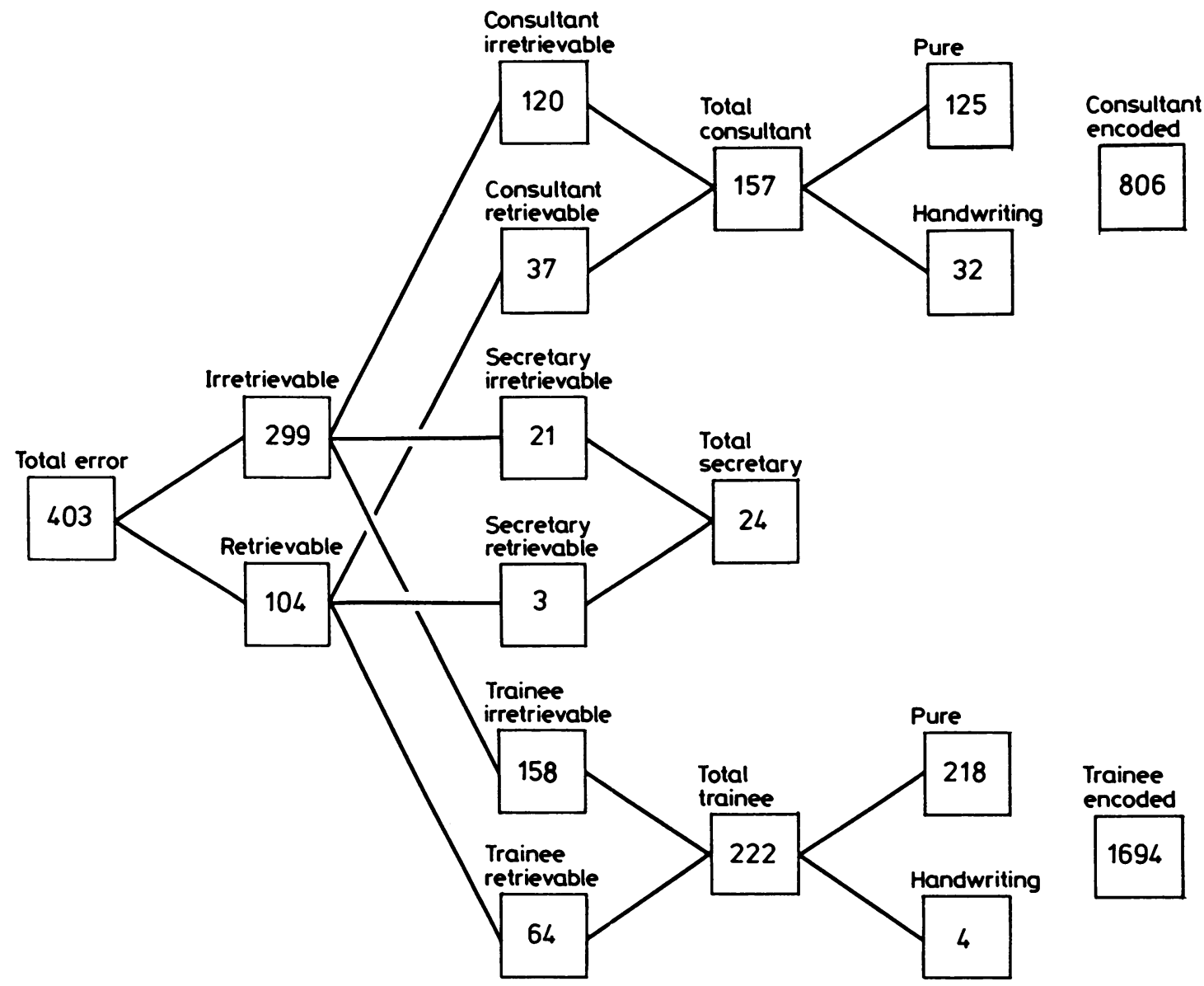

Fig. 1 Crude errors of SNOP coding present in 2500 consecutive surgical reports at the London Hospital and their distribution.

would preclude the case being found by a reasonably diligent researcher in the future." A RETRIEVABLE ERROR was similarly defined as "an error that would not preclude the case being found by a reasonably diligent researcher in the future." The encoding medical staff were classified by the possession of the MRCPath into consultant and trainee. Pure secretarial errors were designated likewise, but those errors that occurred as a consequence of poor handwriting were recorded as medical staff errors.

Statistical analysis of the results was performed using the $\chi^{2}$ test.

\section{Results}

Fig. 1 shows the results from the London Hospital and Fig. 2 those from University College Hospital.
The staffing structure, workload, and broad categories of material handled by the two institutions were sufficiently similar to allow their error to be directly compared.

At the London Hospital $403(16 \cdot 1 \%)$ of the coded reports were considered to be incorrect, while at $\mathrm{UCH}$ $261(10 \cdot 4 \%)$ of the coded reports were erroneous. A similar proportion of the errors were irretrievable at both institutions (London Hospital 299 (74.1\%) compared with UCH $207(79 \cdot 3 \%)$ ). The number of errors attributable solely to the secretarial staff was almost identical in the two institutions (London Hospital 24 $v \mathrm{UCH} 23$ ). Of the 2500 reports coded at each institution, $806(32 \cdot 2 \%)$ were coded by a consultant at the London Hospital compared with a figure of 815 $(32.6 \%)$ at $\mathrm{UCH}$. The percentage of irretrievable errors was similar for both consultants (London Hos- 




Fig. 2 Crude errors in SNOMED coding present in 2500 consecutive surgical reports at University College Hospital and their distribution.

pital $120(76.4 \%)$, UCH $98(78 \cdot 4 \%)$ and trainees $(158$ $(71.2 \%)$ and UCH $84(76.4 \%))$, respectively. If it is accepted that the two departments and the nature of their specimens are similar then the main difference between the two departments is clearly in the contribution of the junior staff, with those at The London making 222 errors and those at UCH making only $110\left(\chi^{2}, p<0.001\right)$. Although there was little difference between the number of errors due to the consultant staff alone in the two hospitals (London Hospital 125 and UCH 116), when handwriting errors were included in the total consultant error the difference became large (157 and 125 , respectively, $\chi^{2}$ $p<0.001$ ). The number of handwriting errors made by trainees was small and had little effect on the difference in the total number of errors by the juniors in the two departments. Seventy six of the total errors in reports coded by trainees at the London Hospital occurred in codes relating to bone marrow disease, $?$ whereas only seven such errors were seen at UCH. If $>$ these errors are removed from the analysis, however, the difference between the trainees in the two institu- $N$ tions remains significant (London Hospital 146 and $^{\circ}$ UCH 110). Errors were distributed throughout othero systems and disease processes with no discerniblew trend.

Because of the comprehensive definition used ino this study, a proportion of the errors might be regarded as trivial. Because of the difficulty in consis-? tently separating a trivial from an important error 0 this was not attempted. It was our impression, how-O ever, that trivial errors occurred at a similar rate ato both institutions and represented less than $10 \%$ of the total. 


\section{Discussion}

The use of numerical coding of data may be of enormous value in pathology and other specialities, and the potential applications of a large body of detailed data are legion. It may form a data base for academic research in a wide variety of fields, allow the recall of material for undergraduate and postgraduate teaching, facilitate patient management, and could form the cornerstone of auditing and departmental management procedures. By the widespread use of a common coding system the regional, national, and international exchange and collation of data could be facilitated. If, however, the stored data are to be of any value they should be easily accessible and accurate. Enlander ${ }^{2}$ undertook a study of manual data coding, using the SNOP system, with residents at Stanford University Hospital where he found that only $76 \%$ of the data were correctly coded. Dinwoodie and Howell ${ }^{6}$ investigated coding errors of hospital discharge and general practice data by professional encoders and found error rates of between $10 \%$ and $20 \%$. Notably, neither the SNOP Sn $^{4}$ or the SNOMED 5 manuals make any mention of the problem of erroneous coding and its potential importance.

The crude error rates at both institutions were high, with more errors being made at the London Hospital $(16.4 \%)$ than at UCH $(10.4 \%)$. The excess of errors at the London Hospital was primarily due to poor coding by the trainee staff, while the consultant staff contributed to the excess by their poor handwriting. If, however, the number of errors due to each group is related to the number of reports that they code then it is clear that at both hospitals the junior staff are substantially more accurate than the senior staff (London Hospital juniors $13.1 \%$ and seniors $19.5 \%$, UCH juniors $6.5 \%$ and seniors $14.2 \%$ )-perhaps because they perform more of the coding. The overall error rate at the two hospitals was $13 \cdot 3 \%$ - that is, four in every 30 reports was incorrectly coded and three of those four were irretrievably lost!

The pattern of errors within each hospital was remarkably similar, with three quarters of all errors being irretrievable and the proportion of irretrievable errors being made by each group (consultant, trainee, and secretary) being similar. The two departments that we studied use identical computer record systems, ${ }^{7}$ have a similar surgical pathological workload, similar proportions of junior and senior staff with similar distribution of responsibilities. The major difference between the two institutions was the coding system used, and we would suggest that the higher error rate at the London Hospital was largely due to deficiencies in the SNOP system. A large proportion of the excess errors at the London Hospital occurred in cases that were difficult to code within the confines of the rather outdated SNOP system, while similar cases could be more easily coded within the more up to date, larger, and more sophisticated SNOMED system. For example, the SNOMED system facilitates the coding of bone marrow disorders, which have proved so difficult using the SNOP system. We suggest that the SNOMED system is superior to the SNOP system, but even with this system at UCH the error rate was $10.4 \%$.

The range of errors seen was wide, but there were several broad groups, all of which were similarly represented at both institutions. Pure secretarial errors comprised a very small proportion of the total $(0.9 \%)$, but they were almost invariably irretrievable errors arising as a result of inversion of topographical and morphological codes, or an inversion, deletion, or addition of numerals within a given code. We had expected a higher pure secretarial error rate, and it is unlikely that this can be much improved, although closer inspection of the codes on the final report by the pathologist may bring the more glaring errors to light. Poor handwriting led to a significant minority of errors $(1 \cdot 1 \%)$ in transcription and this could be improved.

Many of the errors seem to be due to laziness in coding, with failure to consult the appropriate manual and reliance on memory for common codes. The factually correct but unhelpful code was regarded as an error in this study; for instance, the use of "no evidence of tumour" was a common disguise for other specific histological features contained in the written report, such as radiation change in the bladder. Inconsistent coding was noted; examples included the use of codes for either dysplasia or nuclear atypia to describe verbally identical reports of cervical biopsies by the same person. Idiosyncratic coding, in which reports were assigned a personal recall label with no attempt to include specific feature codes, occurred occasionally and exposed the limited foresight of the encoder. Some codes were inexplicable-for example, a lymphoma was coded and assigned the morphological code for lipoma. Finally, it was apparent that many staff at both hospitals failed to appreciate the implications of poor or incomplete coding, and considered it to be a burdensome task. Accuracy and completeness when assigning codes should, we feel, be regarded as an integral part of the surgical report and an important function of the surgical pathologist, either in training or as a consultant.

Various changes could be introduced to reduce the magnitude of error. For example, all members of staff need education in the purpose and scope of data encoding, with particular reference to the system used within their own department. The codes listed in aetiology, function, and morphology should not be regarded as mutually exclusive, and the use of multi- 
ple codes to aid recall should be encouraged. It might be of value for one member of staff to coordinate this and to assume special responsibility for the department (SNOP/SNOMED officer). In addition to the bench manuals, a local coding dictionary or lexicon ${ }^{8}$ could be compiled in the light of intradepartmental experience. Regular audit to assess the error rates should be introduced to maintain standards and identify new problems as they arise.

This error prone and tedious clerical chore may be more appropriately performed by computers, with error rates of less than $5 \%$ of codes being reported. ${ }^{1-36}$ We feel that the problems of manual encoding are such that automatic computer encoding is the optimal method, and any new system should employ such methods in conjunction with SNOMED.

Clearly, these broad conclusions have wider implications, as many other specialities within medicine are rapidly expanding their use of computerised records, and most use some form of numerical coding. It is likely that errors similar to those that we have found are occurring in such systems, and clearly, the value of a data storage system is directly related to the quality of the data that are stored, irrespective of the sophistication of the storage system itself.
We would thank Professors Berry and Isaacson for their encouragement and the medical and technical staff of both departments for their help and cooperation. We gratefully acknowledge the statistical advice and helpful comment of Dr Walter Gregory.

\section{References}

${ }^{1}$ Coles EC, Slavin G. An evaluation of automatic coding of surgical pathology reports. J Clin Pathol 1976;29:621-5.

${ }^{2}$ Enlander D. Computer data processing of medical diagnoses in pathology. Am J Clin Pathol 1975;63:538-44.

${ }^{3}$ Ulirsch RC. Status of anatomic pathology data management systems. Arch Pathol Lab Med 1984;108:884-7.

${ }^{4}$ College of American Pathologists. Systematised nomenclature of pathology (SNOP). Chicago: College of American Pathologists, 1965.

${ }^{5}$ College of American Pathologists. Systematised nomenclature of $\omega$ medicine (SNOMED). Skokie: College of American Pathologists, 1976.

${ }^{6}$ Dinwoodie HP, Howell RW. Automatic disease coding: the "fruit $N$ machine' method in general practice. British Journal of Preventive and Social Medicine 1973;27:59-62.

${ }^{7}$ Swettenham KV, Nickols C, Berry CL. Computer programs in histopathology record keeping. J Clin Pathol 1982;35:40-4.

${ }^{8}$ Gallo P, De Balsi V. A computerised data bank of surgical pathology and cytopathology diagnoses. Virchows Arch Pathol $\overparen{(D}$ Anat 1983;401:345-54.

Requests for reprints to: Dr PA Hall, ICRF Research Fellow, Department of Histopathology, St Bartholomew's Hospital, West Smithfield, London EC1A 7BE, England. 